\title{
Ion acceleration enhanced by additional neutralizing electrons in a magnetically expanding double layer plasma
}

\author{
Kazunori Takahashi ${ }^{a}$ and Tamiya Fujiwara \\ Department of Electrical and Electronic Engineering, Iwate University, Morioka 020-8551, Japan
}

(Received 5 August 2010; accepted 21 September 2010; published online 29 October 2010)

\begin{abstract}
Electrons neutralizing an ion beam are additionally supplied to a magnetically expanding double layer (DL) plasma from the downstream side of the DL. The rf power and the argon gas pressure are maintained at $200 \mathrm{~W}$ and $55 \mathrm{mPa}$, respectively, and the source magnetic field is varied in the range of about 70-550 G. It is observed that the ion beam energy corresponding to the DL potential drop increases up to $30 \mathrm{eV}$ with an increase in the magnetic field when supplying the additional electrons, while it saturates at $20 \mathrm{eV}$ for the case of the absence of the additional electrons. The supplied electrons are believed to be an energy source for the DL such that increasing the magnetic field is able to increase the potential drop beyond the limit found in the absence of the supplied electrons. (C) 2010 American Institute of Physics. [doi:10.1063/1.3499691]
\end{abstract}

Recent studies on low-pressure, magnetically expanding laboratory plasmas containing electric double layers (DLs) have attracted great interest in space plasma and propulsion communities; supersonic ion beams are detected simultaneously with the formation of the DLs in various laboratory experiments using solenoid or permanent magnets. ${ }^{1-5}$ It has been suggested that a DL is the mechanism for several space phenomena involving particle acceleration, ${ }^{6}$ and the DLs are being used in the development of a long-lived electrodeless plasma thruster. $^{7}$

The potential drops $\phi_{\mathrm{DL}}$ of the DLs investigated in the magnetically expanding laboratory plasmas 8,9 and in the diffusion-controlled model ${ }^{10,11}$ treating the expanding magnetic-field effect as a particle loss in the diffusion chamber are three to seven times the electron temperature $\left(\phi_{\mathrm{DL}}\right.$ $\sim 3-7 k_{B} T_{e} / e$ ) for argon plasmas, where $k_{B}, T_{e}$, and $e$ are the Boltzmann's constant, the electron temperature, and the elementary charge, respectively. Then, electron energy distributions observed in the experiment ${ }^{12}$ and in the onedimensional (1D) particle-in-cell simulation ${ }^{13}$ have indicated that the energetic electrons in the source, overcoming the potential drop of the DL and being an energy source for the DL, can neutralize the ion beam accelerated by the DL. These results are consistent with another 1D DL model resembling the sheath theory, ${ }^{14}$ where the effect of the expanding magnetic field is included as a spatial change of the plasma cross section. The model described in Ref. 14 has predicted that the potential drop of the DL cannot exceed $5 k_{B} T_{e} / e$ for argon because of the absence of the energy source for the DL larger than $5 k_{B} T_{e} / e$, i.e., the absence of the neutralizing electrons overcoming the DL for $\phi_{\mathrm{DL}}$ $>5 k_{B} T_{e} / e$.

The recent experiment focused on the role of the magnetic-field strength has reported that the DL is triggered at a threshold of the source magnetic-field strength, ${ }^{15}$ where it has been supposed that the reduction of the plasma loss to the source wall is a key factor in the formation of the DL because the ion Larmor radius is close to the source tube

${ }^{a)}$ Electronic mail: kazunori@iwate-u.ac.jp. radius at the magnetic-field threshold. This fact is also experimentally verified for various diameter source tubes more recently. ${ }^{16}$ Near the field strength threshold, the DL potential drop and the ion beam energy appear to increase with an increase in the field strength around several tens of Gauss (see Fig. 3 in Ref. 15) for a $\sim 14$-cm-diameter source. For higher magnetic field, however, the potential drop saturates and would be limited as predicted by the DL model in Ref. 14. Due to the potential-drop limitation originating from the absences of the neutralizing electrons and the energy source for the DL, the effect of the magnetic field is considered not to be observed for higher magnetic-field strength. Thus, the investigation on the magnetic-field effects is required to be performed under the condition that there are sufficient electrons neutralizing the ion beam and being the energy source for the DL.

In the present letter, we demonstrate that the potential drop of the DL and the energy of the ion beam in the magnetically expanding plasma can be increased by the strong magnetic field under the condition that there are sufficient electrons neutralizing the ion beam downstream of the DL and being the energy source for the DL, where the additional electrons are provided by an electron emitter from the downstream side of the DL. The results imply that the magnetic field has an effect to increase the potential drop of the DL with an increase in the field strength, although this effect did not appear so far in the simple magnetically expanding plasma due to the potential-drop limitation.

Experiments are performed in the electromagnets expanding plasma machine at Iwate University shown in Fig. 1(a). A 35-cm-long and 6.5-cm-diameter cylindrical glass tube (source tube) is connected to a 17.5-cm-long and 20.8$\mathrm{cm}$-diameter grounded stainless steel vacuum chamber (diffusion chamber). The chamber is evacuated to a base pressure below $1 \mathrm{mPa}$. The argon gas is introduced from the source side through a mass flow controller, and the operating argon gas pressure is selected as $55 \mathrm{mPa}$ in the present experiment. An argon plasma is excited by a 7.5-cm-diameter triple-turn loop antenna located at $z=-16 \mathrm{~cm}$ and powered from a $13.56 \mathrm{MHz}$ and $200 \mathrm{~W}$ rf generator, where $z=0$ is 


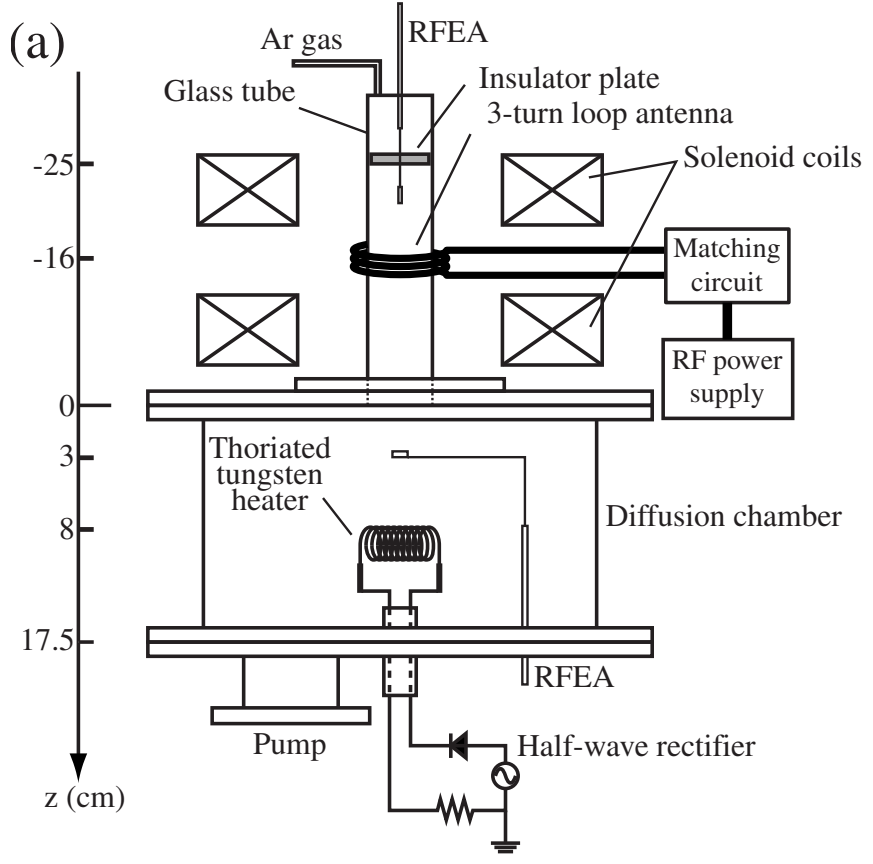

(b)

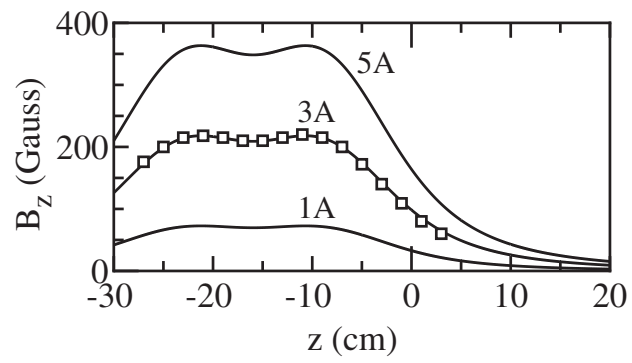

(c)

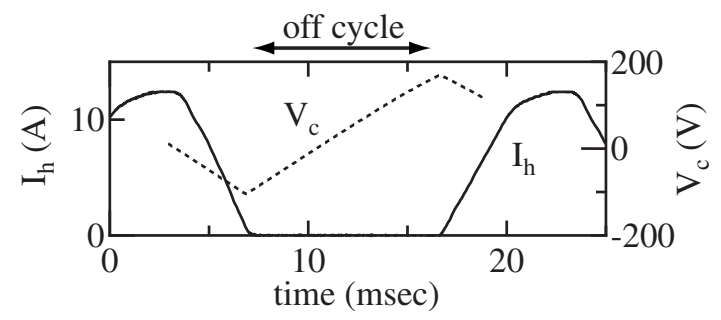

FIG. 1. (a) Schematic of the experimental setup. (b) Calculated magneticfield strength $B_{z}$ (solid lines) for the solenoid current of 1,3 , and $5 \mathrm{~A}$ together with the measured field strength (open squares) for $3 \mathrm{~A}$. (c) Temporal wave patterns of the heater current $I_{h}$ of the electron emitter and the collector bias voltage $V_{c}$ of the RFEA.

defined as the interface between the source tube and the diffusion chamber. Two solenoid coils situated at $z=-8.7 \mathrm{~cm}$ and $z=-23.2 \mathrm{~cm}$ are fed to the currents with the same value and provide expanding magnetic-field configurations shown in Fig. 1(b). Axial profiles of the calculated magnetic-field strength $B_{z}$ for 1,3 , and $5 \mathrm{~A}$ solenoid currents are plotted in Fig. 1(b) as solid lines, together with the measured one for 3 $\mathrm{A}$, where the averaged field strength in the source tube for unit solenoid current is about $70 \mathrm{G} / \mathrm{A}$. The plasma in the source tube is terminated by an insulator plate set at $z=$ $-25 \mathrm{~cm}$, which ensures the current-free condition.

Retarding field energy analyzers (RFEAs) facing radially and axially are set, as shown in Fig. 1(a), to measure a plasma potential and an ion energy distribution function
(IEDF). Under the condition that the gas pressure and the solenoid current are maintained at $55 \mathrm{mPa}$ and $5 \mathrm{~A}$, respectively, the plasma density and the electron temperature measured by a conventional Langmuir probe at $z=3 \mathrm{~cm}$ are about $2 \times 10^{9} \mathrm{~cm}^{-3}$ and $8 \mathrm{eV}$, respectively. Here, we mention that the estimated temperature would be higher than the actual value because the effects of the rf oscillation are not compensated now. ${ }^{17}$ The IEDF proportional to the first derivative of a collector current-voltage $\left(I_{c}-V_{c}\right)$ characteristic of the RFEA can be obtained by a pulsed probe technique. ${ }^{18}$

In order to supply the additional electrons, an electron emitter consisting of 0.25 -mm-diameter thoriated tungsten wires is set at $z=8 \mathrm{~cm}$, which is connected to a half-wave rectifier circuit, where the heater current $I_{h}$ can be monitored through the $0.1 \Omega$ resistor as shown in Fig. 1(c). The collector voltage $V_{c}$ of the RFEA is swept during the off cycle as shown in Fig. 1(c) in order to eliminate the effects of the electric field created by the heater voltage. In the present experiment, the emitter is operated without $\left[I_{h}=0 \mathrm{~A}\right]$ and with $\left[I_{h}\right.$ shown in Fig. 1(c)] electron emission, which are labeled as "heater OFF" and "heater ON," respectively.

The axial profile of the plasma potential $\phi_{p}$ measured by the RFEA facing radially for the case that the electron emitter is not set in the chamber is shown in Fig. 2(a) as crosses, where the argon gas pressure and the solenoid current are set at $55 \mathrm{mPa}$ and $5 \mathrm{~A}$. Near the open end of the source tube, the potential drop of the DL is found to form. The filled circles and the open squares in Fig. 2(a) show the plasma potentials for heater OFF and heater ON, respectively, where the electron emitter is set at $z=-8 \mathrm{~cm}$. It is found that the plasma potential in all regions for heater OFF (ON) is higher (lower) than the result shown as crosses. Since downstream electrons escape into (supplied from) the grounded electron emitter for heater OFF (ON), the sheath voltage at the bottom of the plasma becomes large (small) such that the total charge in the plasma is balanced. However, note that the potential drop of the DL near the open end of the source tube is still observed for both cases. In order to discuss the change in the potential drop of the DL near the source exit due to the additional electrons, the potential differences $\Delta \phi_{p}$ from the upstream potential for heater OFF and ON are plotted in Fig. 2(b) as filled circles and open squares, respectively. Here, $\Delta \phi_{p}$ is defined as $\Delta \phi_{p}(z) \equiv \phi_{p}(z)-\phi_{p}(z=-8 \mathrm{~cm})$. The results clearly present that the potential drop of the DL is enhanced by the additional electrons, where the increase in the potential drop is found to be about 5-6 V.

Simultaneously with the plasma-potential measurements for heater OFF and ON shown in Fig. 2(a), the IEDFs in the axial direction are measured at $z=3 \mathrm{~cm}$ downstream of the DLs by the RFEA facing to the source tube. The experimentally observed IEDFs for heater OFF and ON are plotted as crosses in Figs. 3(a) and 3(b), respectively, together with the Gaussian deconvolutions for each peak (dashed lines) and the combined deconvolutions (solid lines). The IEDFs clearly show two peaks at about (61 and 82 V) in Fig. 3(a) and $(23$ and $50 \mathrm{~V})$ in Fig. 3(b) as indicated by the arrows, where $V_{c}$ yielding the left- and right-side peaks give the local plasma potential $\phi_{p}$ and the beam potential $\phi_{\text {beam. }}$. It is found that $\phi_{p}$ and $\phi_{\text {beam }}$ observed in Figs. 3(a) and 3(b) correspond 

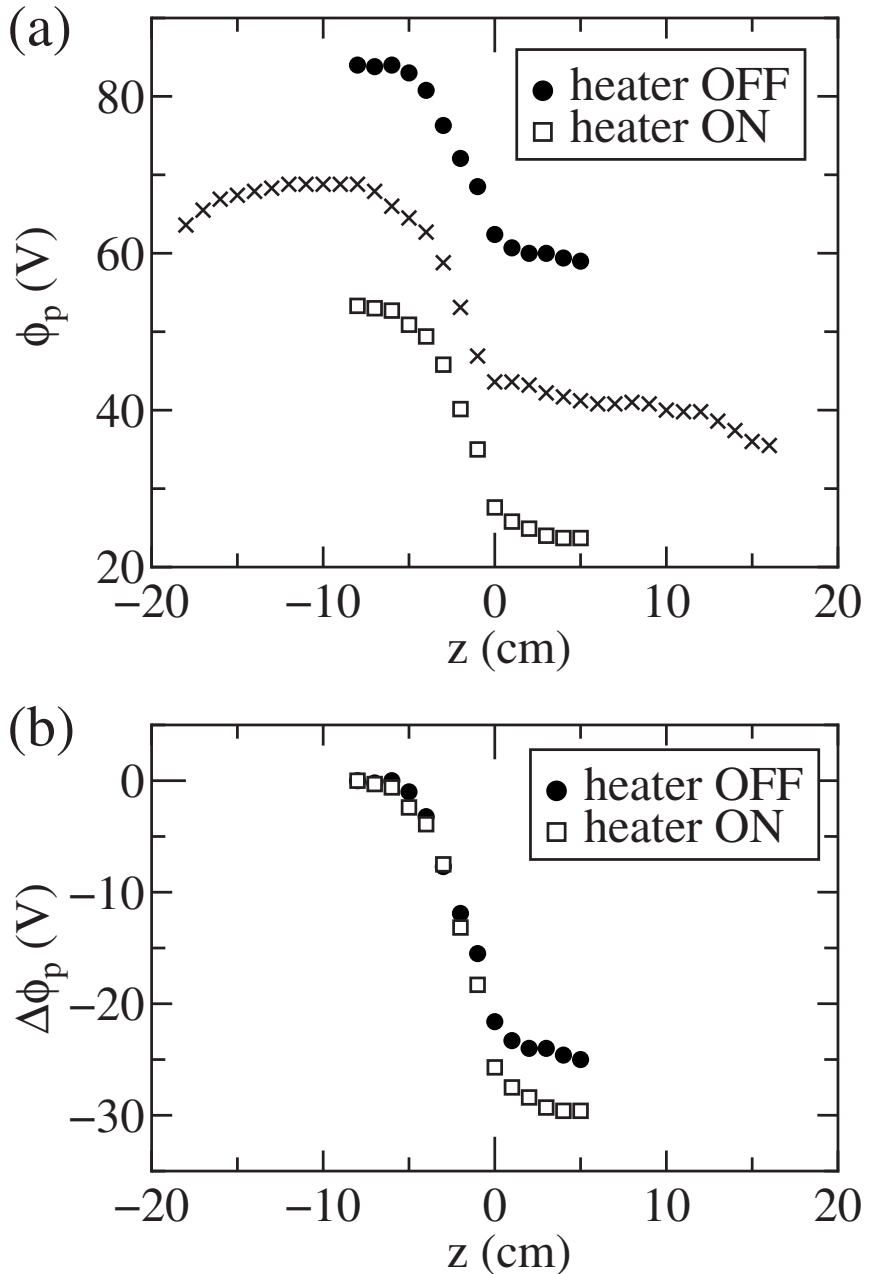

FIG. 2. (a) Axial profiles of the plasma potential $\phi_{p}$ for heater OFF (filled circles) and heater $\mathrm{ON}$ (open squares) for $55 \mathrm{mPa}$, where the crosses are the plasma potential measured without the electron emitter in the chamber. (b) Potential differences $\Delta \phi_{p}$ from the upstream potentials.

to the downstream $(z=3 \mathrm{~cm})$ and upstream $(z=-4 \mathrm{~cm})$ plasma potentials in Fig. 2(a), respectively. Hence, the observed ion beams are accelerated by the potential drops of the DLs near the source exit. As the local plasma potential $\phi_{p}$ corresponds to zero energy in IEDF, the energy $\varepsilon_{\text {beam }}$ of the ion beam can be defined as $\varepsilon_{\text {beam }} \equiv e\left(\phi_{\text {beam }}-\phi_{p}\right)$. The ion beam energies for the heater OFF and $\mathrm{ON}$ are $\varepsilon_{\text {beam }} \sim 21$ and $27 \mathrm{eV}$; the increase in the energy for heater $\mathrm{ON}$ is in good agreement with the change in the potential drop observed in Fig. 2(b).

The ion beam energy, which is the indicator of the potential drop, is measured for various magnetic-field strength, where the solenoid current is changed in the range of 1-8 A (70-560 $\mathrm{G}$ in the source tube). Figure 4 shows the observed ion beam energies $\varepsilon_{\text {beam }}$ for heater OFF (filled circles) and heater ON (open squares) as a function of the solenoid current. The upper axis shows the magnetic-field strength in the source tube $(z=-16 \mathrm{~cm})$ corresponding to the solenoid current shown at the lower axis. The data of the beam energy below 2 A solenoid current are not plotted because the ion beam is not detected for heater OFF, and the ion beam is found to be triggered for the solenoid current above $2 \mathrm{~A}$. The (a)

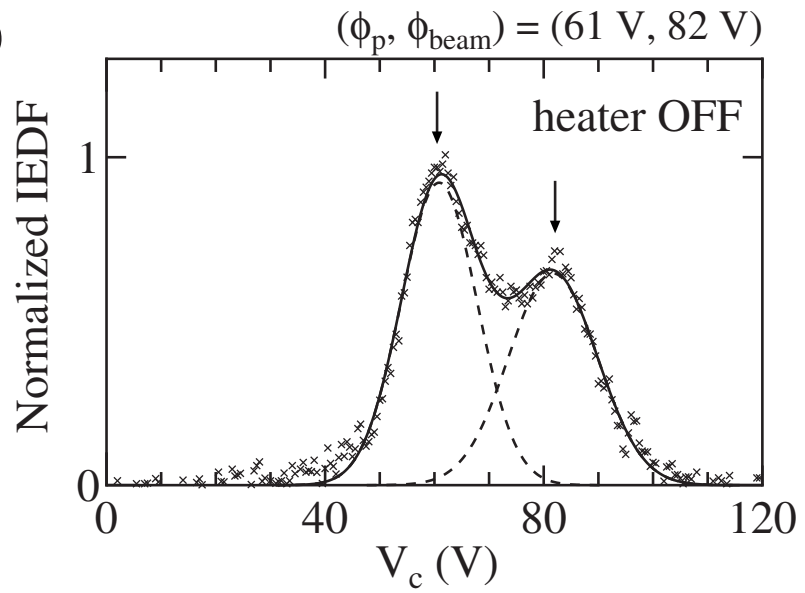

(b)

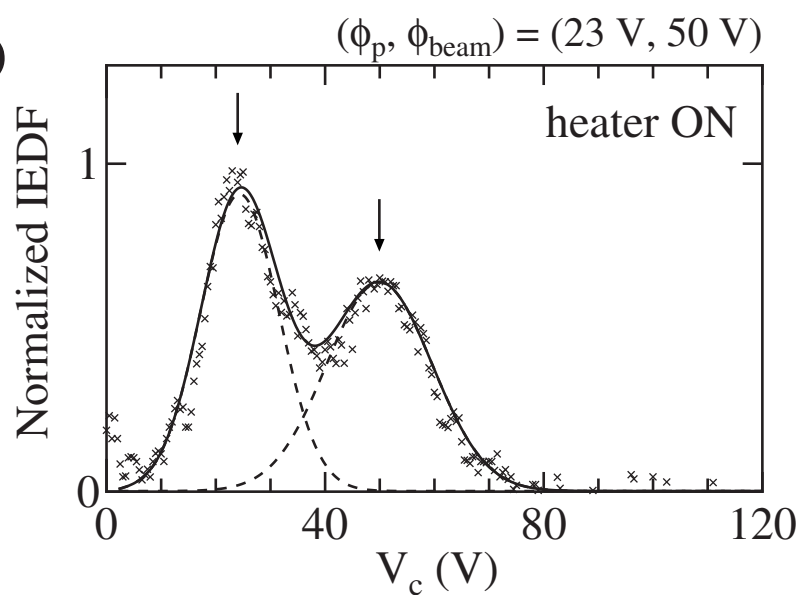

FIG. 3. The IEDFs (crosses) measured at $z=3 \mathrm{~cm}$ for (a) heater OFF and (b) heater $\mathrm{ON}$ for $55 \mathrm{mPa}$, together with the Gaussian deconvolutions for each peak (dashed lines) and the combined deconvolutions (solid lines). The solid arrows show the local plasma potential $\phi_{p}$ and the ion beam potential $\phi_{\text {beam }}$

field strength yielding the appearance of the beam energy is about $140 \mathrm{G}$ in the present experiment, which is a few times the result in the previously reported experiment using the $\sim 14$-cm-diameter source tube in Ref. 15 . At $140 \mathrm{G}$, the Larmor radius of the ions with $0.2 \mathrm{eV}$ temperature ${ }^{19}$ is about 3.3 $\mathrm{cm}$ and is very close to the radius of the present source tube. This result is consistent with the DL characteristics previously reported in a different machine. ${ }^{15,16}$ For heater OFF, it is found that the beam energy increases with an increase in the solenoid current in the range of 2-3 A, while the energy saturates at about $20 \mathrm{eV}$ above $3 \mathrm{~A}$ solenoid current. Although the higher magnetic field would reduce the plasma loss to the source wall and is expected to yield the increase in the potential drop of the DL, the potential drop is considered to be limited by the absence of the electrons neutralizing the ion beam and being the energy source for the DL as mentioned before. Now, our attention is moved to the result for heater ON. Below 3 A solenoid current in Fig. 4, the result for heater ON (open squares) tracks that for heater OFF (filled circles), while above $3 \mathrm{~A}$, the energy is clearly observed to increase up to $30 \mathrm{eV}$ with the increase in the magnetic-field strength. From the results for heater OFF and heater ON shown in Fig. 4, it is found that the expanding 
Upstream magnetic field (Gauss)

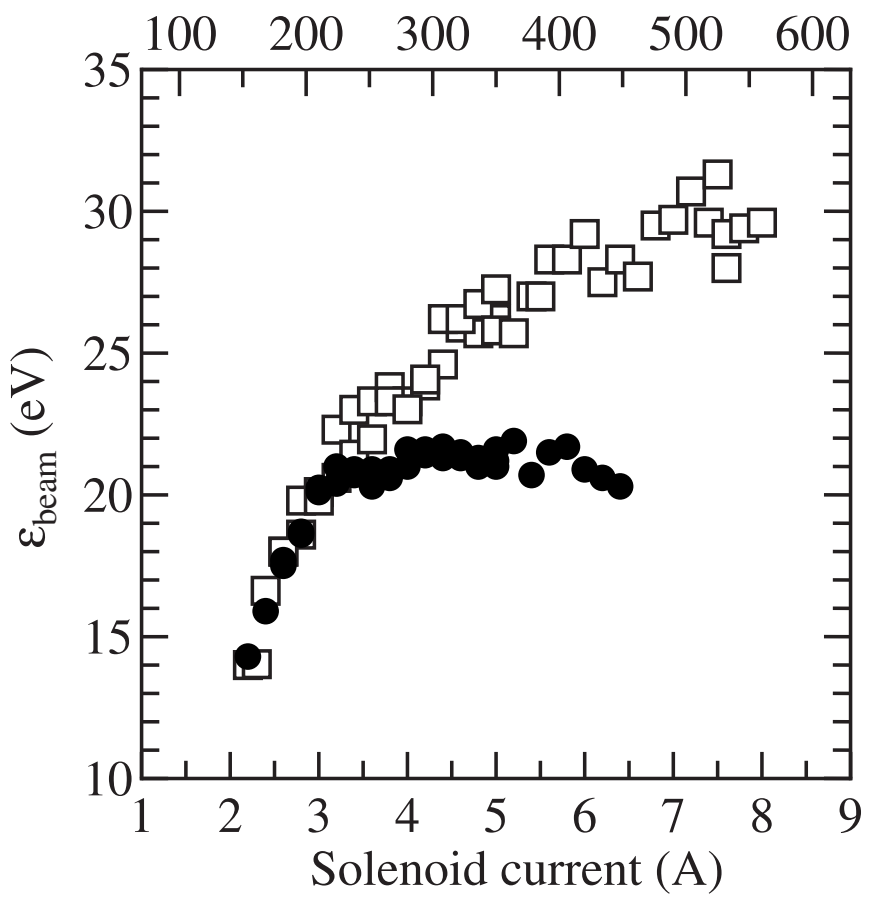

FIG. 4. The observed ion beam energy $\varepsilon_{\text {beam }}$ as a function of the solenoid current for heater OFF (filled circle) and heater ON (open squares) for 55 $\mathrm{mPa}$, where the upper axis shows the magnetic-field strength in the source tube.

magnetic field would basically have effects not only triggering the formation of the DL but also increasing the potential drop of the DL and the ion beam energy with the increase in the field strength. The effect to increase the ion acceleration energy is observed for the first time by supplying the neutralizing electrons additionally in the present experiment (heater ON), although the potential drop is considered to be limited due to the absence of the energy source and the neutralizing electrons as suggested by the 1D DL model ${ }^{14}$ in the simple magnetically expanding plasmas (heater OFF).

Here, we consider the behavior of the additional electrons emitted from the electron emitter in the steady-state DL plasma. In the present experiments, the mean free path for electron-neutral collision is about $80 \mathrm{~cm}$, much longer than the plasma length. Therefore, we can concentrate on the discussion in collisionless plasmas. The electrons emitted from the heater are accelerated by the sheath at the emitter and by the potential drop of the DL while gaining energy and are injected into the upstream source plasma; then, their energy reaches $53.5 \mathrm{eV}$ corresponding to the upstream plasma potential plotted as open squares in Fig. 2(a). The electrons are reflected by the sheath at the upstream floating wall, where the floating wall potential and the potential drop at the floating wall for heater $\mathrm{ON}$ are actually identified as -1 and 54.5 $\mathrm{V}$, respectively, for the same conditions as in Fig. 2 by measuring the floating potential of the Langmuir probe located near the wall; then, the sheath voltage at the upstream wall is large enough to reflect the electrons accelerated by the sheath at the grounded emitter and the DL. After the reflection, they overcome the potential drop of the DL while losing energy and neutralize the supersonic ion beam accelerated by the DL, which is ensured by the upstream insulator plate providing the current-free condition. Hence, the emitted electrons act as both energetic free electrons and electrons neutralizing the ion beam. In this case, the total electron energy contributing to the energy source for the DL would increase because there are additional energetic electrons in the source, which are supplied from the emitter. Based on this consideration, we now suggest that the electrodeless enhancement of the ion acceleration can be achieved by creating the energetic electrons in the source under the strong magnetic field, e.g., by wave heating and so on.

In summary, the potential drop of the DL and the ion beam energy in the magnetically expanding plasma are found to be increased by the presence of the neutralizing electrons and the strong magnetic field. It is demonstrated that the expanding magnetic field has an effect to enhance the potential drop of the DL and the ion acceleration energy with the increase in the field strength under the condition that there are sufficient neutralizing electrons, which would also act as energetic electrons in the source and become the energy source of the DL. Our results would play an important role in the further development of the DL models in the magnetically expanding plasmas. Moreover, the mechanism of the increase in the ion beam energy would also be useful for powering up the thrust force of the electrodeless thruster.

This work was supported by a Grant-in-Aid for Young Scientists (Grant No. A 22684031) from the Ministry of Education, Culture, Sports, Science and Technology, Japan. This work is also partially supported by the TEPCO Research Foundation.

${ }^{1}$ C. Charles, Plasma Sources Sci. Technol. 16, R1 (2007), and references therein.

${ }^{2}$ K. Takahashi, K. Oguni, H. Yamada, and T. Fujiwara, Phys. Plasmas 15, 084501 (2008).

${ }^{3}$ K. Takahashi and T. Fujiwara, Appl. Phys. Lett. 94, 061502 (2009).

${ }^{4}$ S. C. Thakur, Z. Harvey, I. A. Biloiu, A. Hansen, R. A. Hardin, W. S. Przybysz, and E. E. Scime, Phys. Rev. Lett. 102, 035004 (2009).

${ }^{5}$ V. F. Virko, Y. V. Virko, V. M. Slobodyan, and K. P. Shamrai, Plasma Sources Sci. Technol. 19, 015004 (2010).

${ }^{6}$ R. W. Boswell, E. Marsch, and C. Charles, Astrophys. J. 640, L199 (2006).

${ }^{7}$ C. Charles, J. Phys. D: Appl. Phys. 42, 163001 (2009), and references therein.

${ }^{8}$ C. Charles and R. W. Boswell, Phys. Plasmas 11, 1706 (2004).

${ }^{9}$ O. Sutherland, C. Charles, N. Plihon, and R. W. Boswell, Phys. Rev. Lett. 95, 205002 (2005).

${ }^{10}$ M. A. Lieberman and C. Charles, Phys. Rev. Lett. 97, 045003 (2006).

${ }^{11}$ M. A. Lieberman, C. Charles, and R. W. Boswell, J. Phys. D 39, 3294 (2006).

${ }^{12}$ K. Takahashi, C. Charles, R. W. Boswell, T. Kaneko, and R. Hatakeyama, Phys. Plasmas 14, 114503 (2007).

${ }^{13}$ A. Meige and R. W. Boswell, Phys. Plasmas 13, 092104 (2006).

${ }^{14}$ F. F. Chen, Phys. Plasmas 13, 034502 (2006).

${ }^{15}$ C. Charles and R. W. Boswell, Appl. Phys. Lett. 91, 201505 (2007).

${ }^{16}$ K. Takahashi, C. Charles, R. W. Boswell, and T. Fujiwara, Appl. Phys. Lett. 97, 141503 (2010).

${ }^{17}$ I. D. Sudit and F. F. Chen, Plasma Sources Sci. Technol. 3, 162 (1994).

${ }^{18}$ K. Takahashi, Y. Shida, and T. Fujiwara, Plasma Sources Sci. Technol. 19, 025004 (2010).

${ }^{19}$ A. M. Keesee, E. E. Scime, C. Charles, A. Meige, and R. W. Boswell, Phys. Plasmas 12, 093502 (2005). 